\title{
Estudio de la distribución espacial del muérdago enano (Arceuthobium sp.) en el Nevado de Toluca, México, utilizando el Método del SADIE
}

\section{Spatial distribution study of the mistletoe (Arceuthobium sp.) in the EI Nevado de Toluca, Mexico, using the SADIE method}

\author{
Ramírez-Dávila, J. F.1 and E. Porcayo-Camargo2
}

\begin{abstract}
RESUMEN
El Nevado de Toluca fue decretado como Parque Nacional, reconociendo su importancia como sitio de recarga de los acuíferos, así como el valor de sus recursos naturales. Hoy es, sin embargo, escenario de una acelerada depredación tanto humana como natural. El muérdago es una de las plantas parásitas más importantes del grupo de las heterófitas debido a que causa serios perjuicios sobre otros vegetales y que se ha convertido un problema reciente debido a los niveles de infestación del parásito. En este trabajo se obtuvieron datos que permitieron conocer la distribución espacial de este parásito y mapas sobre su densidad en la zona de estudio; para esto se utilizaron plataformas de software ya existentes que han sido probadas ampliamente. El resultado de este estudio permitirá realizar programas de manejo de este parásito que sea sustentada por información confiable, que presumiblemente lleve a lograr un control adecuado de este problema.
\end{abstract}

PALABRAS CLAVE:

Coníferas, heterófitas, muérdago enano, Nevado de Toluca y SADIE.

\begin{abstract}
The Nevado de Toluca was declared a National Park, recognizing its importance as a site for aquifer recharge, as well as the value of its natural resources. Today, however, it is the scene of a rapid depredation both human caused and natural. The mistletoe is one of the most important plant parasites group of heterophytes because they cause serious harm on other plants, which has become a problem recently because of the infestation levels of the parasite. In this study data were obtained that permit to know the spatial distribution of this parasite and maps its density in the study area; they can be used for software platforms already in place that have been tested extensively. The results of this study will permit management programs of this parasite that are broked up by reliable information, presumably leading to pretend adequate control of this problem.
\end{abstract}

KEY WORDS:

Coniferous, heterophyte, mistletoe, Nevado de Toluca and SADIE. Blancas Km. 15 Carr. Toluca-Ixtlahuaca Entronque al Cerrillo. C.P. 50200 Toluca, Méx. Tel. (01 729) 2965531 Ext: 139 Ce: jfrd@uaemex.mx. 


\section{INTRODUCCIÓN}

El Parque Nacional Nevado de Toluca, es una región montañosa que se ubica en la parte central del Estado de México, al suroeste del Valle de Toluca. Según PROTINBOS, tiene un total de 48,571 has de las cuales $77.42 \%$ tiene uso forestal y de esto más de $50 \%$ corresponde a bosques con poca densidad, combinados con usos agrícolas y ganaderos. El Nevado de Toluca o Xinantécatl, según CEPANAF (1972), es un volcán extinto que alcanza una altura de 4558 $\mathrm{msnm}$. El Parque tiene una extensión de 54,000 ha, pobladas por abundante vegetación de pinos, cedros y abetos en donde habitan diferentes especies animales.

Los pinos son un género (Pinus) de árboles 0 , raramente, arbustos, de la familia Pinaceae, subfamilia Pinoideae que presentan una ramificación frecuentemente verticilada y más o menos regular. En México se tiene un gran número de especies diferentes de pinos (género Pinus), mayor que en cualquier otra área en el mundo, de tamaño semejante (Sánchez-González, 2008). Por consecuencia, es un buen lugar para llevar a cabo la observación e interpretación de la variación y evolución de estas especies.

En el Parque Nacional Nevado de Toluca (PNNT) se encuentran dos especies de pino (Pinus montezumae, Pinus hartwegii), las cuales son atacadas por el muérdago enano. El muérdago es una de las plantas parasitas más importantes del grupo de las heterófitas, debido a que causa serios perjuicios sobre otros vegetales a pesar de que se sabe que existe una relación parcial de alimentación entre huésped y hospedero, la que nunca puede ser benéfica y sí a menudo muy dañina para este último (González, 1989). Los muérdagos contienen generalmente clorofila, la cual les permite elaborar algunos de sus propios alimentos a partir de materiales alimenticios inorgánicos tomados de su hospedero (conífera); viven sobre las ramas de árboles $y$ arbustos, siendo tan extraordinaria la aptitud de esta fanerógama para el parasitismo, que se les encuentra sobre hojas y coníferas de todas las alturas y latitudes y en algunos casos produciendo un autoparasitismo en especies de su propia familia (Verduzco, 1976).

En México existen tres especies principales de muérdago enano, Arceuthobium abietis religiosae, el cual es parásito específico de los árboles de oyamel, A. globosum subsp. grandicaule, que es la especie de mayor distribución en la parte central del país y $A$. vaginatum subsp. vaginatum, que es la especie de muérdago más ampliamente distribuida en México (Rodríguez, 1985). Aparentemente no es más que una modesta especie de parásito de las coníferas, pero el muérdago enano posee un sistema único de reproducción que tiene maravillados a los científicos. A diferencia de los ejemplares de otras especies hermanas, cuyas semillas llegan a los árboles después de que un pájaro se las coma y las deposite en el lugar apropiado, este tipo de muérdago lanza su semilla a una distancia que puede llegar a los 20 metros, gracias a una suerte de bomba de agua que se pone en marcha tras un periodo de gestación de 18 meses. Durante todo ese tiempo, la planta acumula el líquido elemento, hasta conseguir la presión necesaria para dispersar sus semillas. Los tallos o brotes aéreos son la parte del parásito que sale fuera del tejido de la hospedera, donde se desarrollan los órganos reproductores, se originan en yemas del sistema endofítico y una vez que éste se ha establecido, están cubiertos por una capa de tejido epidérmico; la formación epidémica original es sustituida por un tejido (epidér- 
mico auricular secundario, pero los tallos no llegan a tener nunca una corteza normal). El muérdago enano puede ser parasitito y vivir indefinidamente sin la emisión de tallos. La presencia de almidón en el sistema endofítico de Arceuthobium sugiere la producción de carbohidratos por estas plantas (Gutiérrez, 1970)

Antes se creía que los muérdagos y hospederas vivían en simbiosis, hoy en día, definitivamente se reconocen las relaciones entre parásito y hospedera; hay sin embargo algo de duda respecto a la transmisión del muérdago hacia su hospedera (Gill y Hawksworth, 1961) citado por Valdivia (1963). La velocidad de intercambio entre hospedera y parásito mediante sustancias coloreadas ha revelado que al paso de éstas es mucho más fácil de la hospedera al parásito que en dirección opuesta, pero no hay información cuantitativa sobre la velocidad del movimiento en cualquiera de las direcciones (Childs y Edgron, 1976).

Las especies del género Arceuthobium requieren para su desarrollo de abundante luz solar, estos requerimientos varían de acuerdo con el desarrollo mismo de la planta. La abundancia de luz solar, condición que se presenta en montes aclarados, es sumamente favorable para el desarrollo y diseminación del parásito, los brotes relacionados con vigor y abundancia en su presencia, mayormente cuando se encuentran infestando árboles residuales en áreas que han sido explotadas y donde el estrato inferior de la vegetación está formado por renuevo sobre el cual cae la semilla del parásito (Korstian, 1922) citado por Valdivia (1963) y Gutiérrez (1970).

La edad del árbol tiene una importancia muy grande respecto a la susceptibilidad a ser infestado, árboles viejos son menos susceptibles por la resistencia que presenta su corteza engrosada a la penetración de la radícula de la semilla en germinación y por la carencia de tejido clorofiláceo expuesto. La susceptibilidad de una planta a ser parasitada disminuye proporcionalmente con su crecimiento, alcanzando un máximo aquéllas que tienen un diámetro de 10 a $15 \mathrm{~cm}$ (Gill y Hawksworth, 1961). La variación y altitud dentro de la cual crece este parásito en México, según Gill y Hawksworth, (1961) es de los 2,850 hasta $3,150 \mathrm{msnm}$. No hay una selectibilidad marcada con respecto a la exposición, encontrándose el muérdago en sitios de cualquier exposición. Las infestaciones del muérdago se desarrollan en pendientes moderadas como pronunciadas y los cambio bruscos de temperatura no afectan al muérdago enano (Gill y Hawksworth, 1961)

Varias pináceas son fácilmente infestadas por muérdago enano, ocasionándoles debilitamientos muy considerables que repercuten en su crecimiento e incremento. El primer signo visible de la infestación del muérdago es un ligero abultamiento causado por el estímulo local de la corteza, floema y xilema de la hospedera (Vega, 1976). A medida que transcurre el tiempo y el sistema endofítico crece dentro de la hospedera, la rama puede distorsionarse formando a veces "escobas de brujas". Por regla general estas ramas infestadas sobreviven varios años a las que están libres del parásito, absorbiendo gran cantidad de nutrientes que normalmente irían hacia la parte superior de la copa de los árboles; estas ramas atrofiadas con hábitos de crecimiento anormal son de follaje muy denso y alcanzan un diámetro superior a los demás (Gutiérrez, 1970). El muérdago enano reduce el vigor del árbol, lo que trae como consecuencia una reducción en el crecimiento longitudinal que puede llegar a un $30-40 \%$ y el crecimiento en diámetro puede reducirse en un $40 \%$ en árboles altamente infestados (Hawks- 
worth, 1961). Otra característica del ataque del muérdago es que predispone a los árboles infestados a un ataque intenso de descortezadores y barrenadores, pudrición de la madera por presencia de algunos hongos, enfermedades de la raíz, así como debilitamiento en el tronco y ramas que llegan a sufrir fácil rompimiento y caídas por el viento, y como consecuencia al caer árboles y ramas muertas son material propicio para incendios (Vega, 1976).

Los principales problemas de las coníferas en ladera norte del Parque Nacional Nevado de Toluca (cauce del Lerma) son la deforestación, los incendios, las plagas y enfermedades. Dentro de estas últimas las crecientes poblaciones de muérdago enano se han convertido en un serio problema sanitario para los bosques de coníferas de la región.

Los daños que causa el muérdago enano a los árboles son los siguientes: a) hipertrofia del fuste y de las ramas en el punto infestado; b) atrofia y posterior rotura del fuste y de las ramas de las partes infestadas hacia la cúspide debido al peso del parásito, de manera que los puntos del sujeto parasitado fácilmente terminan en penachos de muérdagos; $c$ ) produce la deformación de las ramas de los árboles afectados al culminar el ataque; d) mata de modo rápido principalmente el arbolado joven, retarda grandemente el crecimiento tanto en altura como en diámetro de los árboles de mayor edad; e) interfiere seriamente en la vida normal del árbol, provocándole a la postre la muerte al adquirir frondosidad el parásito y por consiguiente aumentar sus necesidades de sostén y nutrientes; f) al inicar la primavera, época de mayor crecimiento de los árboles, se retardan las funciones nulificando el desarrollo, altura y diámetro del sujeto, dándole a la postre una apariencia de achaparramiento; $g$ ) al debilitar grandemente a los árboles, éstos quedan predispuestos al ataque de insectos y microorganismos; $h$ ) en aquellos lugares donde se han aplicado cortas como medida de control sobre los árboles intensamente atacados por el muérdago enano, pero que se han dejado algunos sujetos con parasitismo incipiente, viene una propagación extraordinaria del muérdago enano como consecuencia del aclareo que sufrió la masa y la mayor cantidad de luz que reciben estas plantas; i) reduce considerablemente la producción de semillas fértiles de los sujetos atacados y en muchas ocasiones llega a nulificarla totalmente.

Como se aprecia, los daños causados por las poblaciones cada vez más alarmantes de muérdago enano en la región, han provocado un impacto negativo creciente sobre las poblaciones de coníferas en la zona del cauce del Balsas. Dicho impacto negativo ha sido observado pero no cuantificado hasta la fecha, hecho que ahora resulta de vital importancia para poder tomar medidas de control.

\section{OBJETIVOS}

Establecer el tipo de distribución espacial de las poblaciones de muérdago enano, parásitos de coníferas, en la ladera norte (cuenca del Lerma) del Parque Nacional Nevado de Toluca.

Modelizar la distribución espacial de las poblaciones de muérdago enano mediante la utilización de las estadísticas no espacial (índices de dispersión y distribuciones estadísticas) y espacial, con el Análisis Espacial por Índices de Distancia (SADIE). 
Establecer la existencia de una estabilidad espacial y temporal de las poblaciones del muérdago enano.

\section{MATERIAL Y MÉTODOS}

El presente trabajo se realizó en el Parque Nacional Nevado de Toluca que está ubicado en el Estado de México, en los municipios de Zinacantepec, Toluca, Calimaya, Coatepec de Harinas, Tenango del Valle, Villa Guerrero, Almoloya de Juárez, Temascaltepec y Amanalco de Becerra; y situado en las coordenadas UTM con 2102398 y los 2134700 de metros Norte y 399833 y los 432253 de metros Este. El Parque Nacional Nevado de Toluca es uno de los principales parques, ya que ocupa el primer lugar en su extensión, siendo su superficie de 53913 hectáreas, ocupando $52 \%$ de los diez parques del Estado de México.

Para el estudio del comportamiento espacial del muérdago enano se establecieron en la ladera norte (cuenca del Lerma) del PNNT 10 parcelas experimentales de coníferas, de una hectárea cada una. Cabe señalar que cada una de las parcelas estaba situada a diferentes altitudes sobre el nivel del mar. Este estudio se realizó durante el año 2006. Se estableció una malla rectangular de una hectárea de diámetro y se muestreó a cada 10 metros, en ambas direcciones, alrededor de 100 árboles por malla, cada árbol se georeferenció utilizando un DGPS para obtener sus coordenadas espaciales, además se realizó un conteo de números de muérdagos en cada árbol muestreado y su identificación correspondiente. Se realizó una exploración estadística de los datos originales de las poblaciones de muérdago enano para cada muestreo. Con base en lo anterior fue necesario la realización de una transformación logarítmica de los datos $\left[\log _{10}(n+1)\right]$ para normalizarlos.

\section{Estadística clásica}

Existen dos métodos generales para establecer la distribución espacial de los organismos (Binomial Negativa y Poisson) y los índices de dispersión (de dispersión y de Green); ambos se utilizaron en este estudio para realizar la comparación pertinente entre lo hallado con la estadística no espacial y la estadística espacial. Para todas las distribuciones estadísticas se utilizó el programa MLP de máxima verosimilitud (Ross, 1987) para ajustar los modelos a los datos obtenidos. La bondad del ajuste fue examinada con un test c ${ }^{2}$ (Sokal y Rohlf, 1995).

\section{Estadística espacial}

Análisis Espacial por Índices de Distancia (SADIE)

El objetivo de SADIE es establecer el modelo espacial de una población muestreada midiendo la distancia a la cual pueden desplazarse los individuos de la muestra observada. Perry y Hewitt (1991) desarrollaron un índice que utilizaba los datos colectados dentro de una cuadrícula ya preestablecida antes del muestreo, determinando que era un índice biológicamente más descriptivo e informativo de la distribución espacial de las poblaciones de insectos que el índice de dispersión, el cual depende directamente de la abundancia de la población. Perry (1995a) indicó que para datos recolectados en ubicaciones específicas, el uso de la distancia para la regularidad es muy adecuado. Demostró cómo distinguir no aleatoriedad en la forma de heterogeneidad estadística, de la no aleatoriedad espacial. Perry (1995b) desarrolló y extendió el uso del índice de la distancia para la regularidad $\left(\mathrm{I}_{\mathrm{a}}\right)$ para el establecimiento de la estructura espacial de las poblaciones de insectos. Además, introdujo dos diagramas de diagnóstico como ayuda a la interpretación y un índice 
nuevo para estimar el número de focos de agrupamiento de una población, el índice $J_{a}$. Además, señaló que la escala de muestreo es un determinante importante del patrón espacial total. Alston (1996) corroboró que la distancia para la regularidad $(D)$ proporciona bases más adecuadas para elaborar un índice, ya que el índice de agrupamiento desarrollado por Perry y Hewitt (1991) tiene serias limitaciones para detectar múltiples grupos, amontonamientos o parches. En el presente trabajo se utilizaron el índice basado en la distancia para la regularidad $l_{a}$ y el índice $J_{a}$, basado en la distancia del agrupamiento Perry (1995a,b) para establecer el modelo de distribución de las poblaciones de muérdago enano.

\section{Estimación de los índices $I_{a}$ y $J_{a}$}

Los datos recolectados en una cuadrícula prediseñada (conformada por unidades de muestreo), se consideran como un sistema de conteo de individuos, donde $\mathrm{i}=1, \ldots, \mathrm{n}$ unidades de muestreo. Se conoce además, la posición bidimensional $\left(\mathrm{x}_{\mathrm{i}}, \mathrm{y}_{\mathrm{i}}\right)$ de cada unidad de muestreo y su conteo asociado, $\mathrm{N}_{\mathrm{i}}$. La distancia para la regularidad, $D$, es el valor mínimo de la distancia total que los individuos en la muestra pueden haberse movido, de una unidad de muestreo a otra, de modo que todas las unidades de muestreo contuvieran un número idéntico de individuos. La solución se refiere a la manera óptima en la cual los individuos se moverían desde cada unidad de muestreo con un conteo inicial más grande que la media, a otras unidades de muestreo con un conteo inicial más pequeño que la media. Si entonces los conteos observados se permutan aleatoriamente entre las unidades de muestreo, de modo que la muestra que resulta sea un cambio o reajuste simple de la original, entonces $\mathrm{P}_{a}$ (probabilidad de agregación) representa la proporción de muestras seleccionadas al azar con distancia para la regularidad tan grande como, o más grande que, el valor observado, $D$. Intuitivamente, con un valor grande de $D$ podría esperarse que estuviera implicado un agregamiento o un agrupamiento, por ejemplo, un patrón espacial heterogéneo, e inversamente, un valor pequeño de $D$ implicaría una regularidad, por ejemplo, un patrón espacial uniforme. Un valor de $P_{a}$ derivado de un número suficientemente grande de aleatorizaciones proporciona una prueba formal de aleatoriedad; la hipótesis nula de aleatoriedad espacial se puede rechazar, si $P_{a}<0,025$ (en favor de una hipótesis alternativa de agregación), o si $P_{a}>0,975$ (en favor de la alternativa de regularidad) dando el usual $5 \%$ de probabilidad de rechazar la hipótesis nula cuando es verdad. Si la distancia media aritmética para la regularidad de las muestras aleatorias se denota como $E_{\mathrm{a}}$, entonces el índice de agregación, denominado $l_{a}$, está definido como $l_{a}=D / E_{a}$. Usualmente se dice que una muestra es agregada si $I_{a}>1$, la muestra es espacialmente aleatoria si $l_{a}=1$, y la muestra es regular si $I_{a}<1$. Un número total de 2000 aleatorizaciones son suficientes para derivar los valores de los índices correspondientes.

El término $C$ denota la distancia para el agrupamiento, que es el valor mínimo de la distancia total que los individuos de la muestra deben moverse para congregarse en una unidad. Este valor se encuentra más pronto que $D$, usando una búsqueda directa simple sobre todas las unidades de muestreo; a la unidad de muestreo con el valor mínimo se le denomina como el "foco" del agrupamiento. Permutaciones aleatorias de los conteos observados conducen a una proporción denominada $Q_{a}$ (probabilidad de agrupamiento), con una distancia para el agrupamiento tan pequeña, o más pequeña que el valor observado, $C$. Intuitivamente, para datos que comprenden un agrupamiento individual, un valor pequeño de $C$ denotaría un patrón espacial agregado; 
inversamente un valor alto de $C$ implicaría un patrón espacial regular. Análogamente, la hipótesis nula de aleatoriedad puede ser rechazada si $Q_{a}<0,025$ (en favor de la alternativa de agregación) o si $Q_{a}>0,975$ (en favor de la alternativa de regularidad), y si la distancia media para el agrupamiento para muestras aleatorias se denota como $F_{a}$, entonces el índice de agregación $J_{a}$, se define como $J_{a}=F_{a} / C$. Como en el caso del índice $l_{a}$, valores de $J_{a}>1$ usualmente indican una muestra agregada, $J_{a}=1$ representan datos espacialmente aleatorios y $J_{a}<1$ muestras regulares. De esta manera, los valores del índice $J_{a}$ sirven para corroborar los resultados obtenidos con el índice $\mathrm{I}_{\mathrm{a}}$. Además, este índice se utiliza para discriminar entre patrones espaciales donde hay un único agrupamiento importante para el cual sus valores son significativamente mayores que la unidad, y en donde hay dos o más agrupamientos para los cuales su valor no es significativamente diferente de la unidad o incluso menor que ella. Para determinar la significación con respecto a la unidad se utiliza su probabilidad respectiva $\left(Q_{a}\right)$ (Perry, 1998). Los valores de $I_{a}$ y $J_{a}$ para conteos aleatorios no están correlacionados, por lo que se pueden utilizar también 2000 aleatorizaciones en el software empleado para obtener sus respectivos valores. El programa utilizado en este trabajo para determinar los valores y las probabilidades de ambos índices fue el SADIE 1.22 (programa cedido por el doctor Perry). Se utilizaron mapas de densidad realizados con antelación mediante un análisis geoestadístico (Samper y Carrera, 1990) en el cual se empleó el Krigeado Ordinario*, para corroborar los resultados obtenidos con los índices del SADIE.

Método de interpolación desarrollado por el geólogo sudafricano D. G. Krige.

\section{Cálculo de la superficie infestada con base en mapas de densidad}

Con base en mapas realizados con antelación utilizando el método del Krigeado Ordinario, se determinó el porcentaje de infestación establecido por el parásito en cada una de las parcelas experimentales. Para realizar lo anterior, se utilizó el programa Surfer 9.0.

\section{Estabilidad espacial y temporal}

Se compararon los mapas elaborados con anterioridad con el método del Krigeado Ordinario, con la finalidad de establecer si existía o no una estabilidad espacio-temporal de las poblaciones del muérdago enano. La comparación para establecer la estabilidad espaciotemporal de los mapas citados se llevó a cabo mediante el Índice de Asociación del SADIE llamado $I_{m}$ (Perry y Klukowsky, 1997 y Korie et al., 2000). Si $I_{m}>0$, indica que existe una asociación o una estabilidad espacio-temporal entre los mapas sí existe un nivel de significación de $P_{m}<0.025$. La determinación de la estabilidad se realizó con el SADIE 1.22.

\section{RESULTADOS}

Con los datos obtenidos fue posible generar la modelización y mapeo del comportamiento espacial del muérdago enano. Se estableció el porcentaje de infestación en cada una de las parcelas de estudio y se logró determinar si existía estabilidad espacio-temporal de este parásito en las parcelas estudiadas, y con ello se consiguió determinar realmente el patrón de comportamiento espacial presente por parte del parásito. Los resultados obtenidos en cada una de las 10 parcelas de estudio de una hectárea en la ladera norte (cuenca del Lerma) del Parque Nacional Nevado de Toluca, se detallan enseguida: 
El objetivo general planteado en este estudio era modelizar y generar mapas de la distribución espacial del muérdago enano, parásito de coníferas, del Parque Nacional Nevado de Toluca. Para tal hecho se utilizaron dos métodos que permitieron establecer dicho comportamiento espacial. Además, se realizó una comparación entre los resultados de las parcelas, de tal manera que dicha actividad permitiera conocer con mayor precisión la distribución espacial de este parásito y al mismo tiempo se establecieran las ventajas y desventajas de cada método empleado.

Se realizaron apartados específicos de los resultados alcanzados con cada uno de los métodos utilizados y de esta manera dejar más claro qué se logró alcanzar con cada uno de ellos.

\section{Distribución espacial del muérdago enano}

\section{Estadística No Espacial}

En el Tabla 1 se observa el resumen estadístico del número de muérdagos enanos presentes en los árboles de coníferas muestreados. Se logró determinar gracias al Coeficiente de Curtosis que no existía normalidad en los datos recolectados, por lo tanto fue necesario realizar una transformación de los mismos para poder utilizar los datos en la estadística no espacial y en el análisis. Para llevar a cabo esto se utilizó una transformación logarítmica de los mismos $\left[\left(\log _{10}(n+1)\right]\right.$, una vez realizado este proceso se constató que los datos ya se distribuían de formal normal aplicando nuevamente el Coeficiente de Curtosis. Dicho coeficiente indica normalidad cuando registra valores entre -3 y 3 .

El intervalo de densidad de muérdagos enanos por conífera varió entre 4,282 y 1,472 . Las parcelas con mayor densidad de muérdagos (3 y 10) se encuentran ubicadas a altitudes más bajas que las otras tres, habría que determinar si la altitud en realidad juega algún papel importante para determinar la densidad de este parásito, esto no se realizó en este estudio, pero sería muy interesante corroborarlo en estudios posteriores. En este ambiente hetero-

Tabla 1. Resumen estadístico del parásito muérdago enano.

\begin{tabular}{lccccc}
\hline PARCELAS & $X$ & $S$ & $S^{2}$ & $\begin{array}{c}\text { COEFICIENTE } \\
\text { DE CURTOSIS (1a)DE CURTOSIS }\left(2^{\text {a }}\right)\end{array}$ \\
\hline P1 & 3,727 & 3,981 & 15,851 & 4,471 & 1,085 \\
P2 & 3,381 & 3,440 & 11,834 & 6,983 & $-1,902$ \\
P3 & 4,282 & 2,934 & 8,608 & 10,627 & 0,441 \\
P4 & 3,300 & 3,333 & 11,111 & 19,552 & 0,833 \\
P5 & 2,300 & 2,395 & 5,735 & 16,561 & $-1,953$ \\
P6 & 1,472 & 2,285 & 5,224 & 6,381 & 1,267 \\
P7 & 1,818 & 3,059 & 9,361 & 10,772 & 0,821 \\
P8 & 2,300 & 3,520 & 12,395 & 5,222 & $-0,661$ \\
P9 & 3,281 & 6,175 & 6,175 & 16,192 & 1,993 \\
P10 & 4,036 & 7,332 & 53,760 & 19,356 & 0,240 \\
\hline
\end{tabular}


géneo donde se ubicaron las distintas parcelas y en donde se realizaron por tanto los diferentes muestreos, era de esperarse que existiera una considerable variabilidad de la repartición espacial de este parásito. La varianza de los datos se halló entre 53,760 y 5,224.

En cuanto al tipo de distribución espacial de los muérdagos enanos encontrada en cada una de las parcelas experimentales, los resultados se hallan registrados en la tabla 2 . El índice de dispersión señala que en todas las parcelas se encontró una distribución agregada del muérdago enano, con excepción de las parcelas 4, 6 y 9, donde indica una distribución aleatoria. Por su parte, el índice de Green confirmó dicha agregación, pero en este caso para todas las parcelas, indicando, en función de su bajo valor (Tabla 2), que la agregación era leve. Estos datos un tanto contradictorios señalan algunas de las limitaciones de estos métodos para determinar con precisión la distribución espacial del parásito.
Por otro lado, con base en las distribuciones estadísticas, los resultados indican que en las parcelas 1, 2, 4, 5, 6, 7, 9 y 10 se ajustó una distribución binomial negativa (agregación) a los datos. En la parcelas 2, 5 y 6 también se efectuó un ajuste a la distribución de Poisson (aleatoria), por lo que en este caso no se puede discernir la real distribución espacial del muérdago enano en estas parcelas, ya que no es posible que el parásito tenga al mismo tiempo dos tipos de distribución espacial. Quizás la explicación a dicha dualidad puede estar en que para valores altos del parámetro $k$, como es relativamente el caso, la Binomial Negativa tiende a la distribución de Poisson, por lo que al realizar el ajuste estadístico por máxima verosimilitud se ajusten a los datos ambos modelos indistintamente. Los resultados obtenidos por el índice de dispersión y el índice de Green tampoco dejan clara la situación, ya que en los dos primeros casos indican una agregación y el caso de la parcela 6 el índice de dispersión indica aleatoriedad

Tabla 2. Índices de dispersión y distribuciones estadísticas del parásito muérdago enano.

\begin{tabular}{lccccc}
\hline PARCELAS & $\begin{array}{c}\text { ÍNDICE DE } \\
\text { DISPERSIÓN }\end{array}$ & $\begin{array}{c}\text { ÍNDICE DE } \\
\text { GREEN }\end{array}$ & POISSON & $\begin{array}{c}\text { BINOMIAL } \\
\text { NEGATIVA }\end{array}$ & $K$ \\
\hline P1 & $2,32^{\mathrm{s}}$ & 0,002 & $\mathrm{NS}$ & $\mathrm{S}$ & 1,62 \\
P2 & $1,56 \mathrm{~s}$ & 0,003 & $\mathrm{~S}$ & $\mathrm{~S}$ & 6,92 \\
P3 & $3,22^{\mathrm{s}}$ & 0,004 & $\mathrm{NA}$ & $\mathrm{NA}$ & - \\
P4 & $0,77^{\mathrm{ns}}$ & 0,001 & $\mathrm{NS}$ & $\mathrm{S}$ & 4,73 \\
P5 & $4,89 \mathrm{~s}$ & 0,005 & $\mathrm{~S}$ & $\mathrm{~S}$ & 9,73 \\
P6 & $0,86 \mathrm{~ns}$ & 0,001 & $\mathrm{~S}$ & $\mathrm{~S}$ & 11,35 \\
P7 & $2,71^{\mathrm{s}}$ & 0,003 & $\mathrm{NS}$ & $\mathrm{S}$ & 5,22 \\
P8 & $3,39 \mathrm{~s}$ & 0,006 & $\mathrm{NA}$ & $\mathrm{NA}$ & - \\
P9 & $0,65^{\mathrm{ns}}$ & 0,004 & NS & $\mathrm{S}$ & 2,80 \\
P10 & $3,02^{\mathrm{s}}$ & 0,007 & NS & S & 5,65 \\
\hline
\end{tabular}


y el de Green, agregación. Esto demuestra nuevamente las contradicciones en las que pueden caer estos métodos y, por lo tanto, señalan sus limitaciones.

Los datos obtenidos en las parcelas 3 y 8 no se pudieron ajustar a ninguna distribución estadística, ya que no fue posible obtener la convergencia de los algoritmos de ajuste por máxima verosimilitud. Esto es una muestra más de las limitaciones de los métodos de la estadística no espacial.

\section{Estadística Espacial}

\section{Análisis Espacial por Índices de Distancia (SADIE)}

Los datos obtenidos con la aplicación de los índices del SADIE se concentran en la tabla 1, en ella se observa que en el caso del índice $\mathrm{I}_{\mathrm{a}}$ el valor más alto se registró en la parcela 2 , que fue de
1,76 y el más bajo en la parcela 10, igual a 1,27 . En todos los casos el índice $l_{a}$ fue significativamente superior a 1 (Tabla 3 ) lo cual señala una distribución agregada.

En lo referente al índice $\mathrm{J}_{\mathrm{a}}$ el valor mayor se dio en la parcela 2, que fue de 1,25 y el más bajo en la parcela 7 , con un valor de 1,07. El índice $J_{a}$ en todas las parcelas experimentales también fue superior a la unidad, este dato confirma la agregación detallada en el índice anterior.

Con los datos obtenidos con el índice $\mathrm{J}_{a}$, fue posible establecer la cantidad de centros de agregación que se hallaron en cada una de las parcelas estudiadas. En cuanto a este dato, el valor del índice $J_{a}$ en todas las parcelas no fue significativamente superior a 1 , lo que permite determinar que la distribución espacial de las poblaciones del parásito se concentraba en diferentes centros de agregación.

Tabla 3. Valor de los Índices $\mathrm{I}_{\mathrm{a}} \mathrm{y} \mathrm{J}_{\mathrm{a}} \mathrm{y}$ sus respectivas probabilidades $\mathrm{P}_{\mathrm{a}}$ y $\mathrm{Q}_{\mathrm{a}}$ en la población de muérdago enano.

\begin{tabular}{lcccl}
\hline Parcelas & $\mathrm{l}_{\mathrm{a}}$ & $\mathrm{P}_{\mathrm{a}}$ & $\mathrm{J}_{\mathrm{a}}$ & $\mathrm{Q}_{\mathrm{a}}$ \\
\hline P1 & 1,66 & $0,007^{\mathrm{s}}$ & 1,21 & $0,241^{\mathrm{ns}}$ \\
P2 & 1,76 & $0,015 \mathrm{~s}$ & 1,25 & $0,274 \mathrm{~ns}$ \\
P3 & 1,32 & $0,003^{\mathrm{s}}$ & 1,15 & $0,162^{\mathrm{ns}}$ \\
P4 & 1,47 & $0,010 \mathrm{~s}$ & 1,17 & $0,205 \mathrm{~ns}$ \\
P5 & 1,70 & $0,002 \mathrm{~s}$ & 1,23 & $0,196 \mathrm{~ns}$ \\
P6 & 1,51 & $0,008 \mathrm{~s}$ & 1,11 & $0,254^{\mathrm{ns}}$ \\
P7 & 1,47 & $0,012 \mathrm{~s}$ & 1,07 & $0,286 \mathrm{~ns}$ \\
P8 & 1,30 & $0,004 \mathrm{~s}$ & 1,09 & $0,128 \mathrm{~ns}$ \\
P9 & 1,55 & $0,009 \mathrm{~s}$ & 1,16 & $0,216^{\mathrm{ns}}$ \\
P10 & 1,27 & $0,017 \mathrm{~s}$ & 1,19 & $0,249 \mathrm{~ns}$ \\
\hline
\end{tabular}


Cálculo de la superficie infestada con base en mapas de densidad

Lo detallado con los índices del SADIE se observa con precisión en los mapas de densidad elaborados con este programa.

Los mapas elaborados con el método del Krigeado Ordinario se detallan en la figura 1. La distribución agregada de las poblaciones del parásito queda de manifiesto en cada uno de ellos, lo que corrobora lo establecido por los índices $\mathrm{l}_{\mathrm{a}} \mathrm{y} \mathrm{J}_{\mathrm{a}}$.

En los mapas realizados se alcanzan a apreciar los diferentes focos de agregación o de agrupamiento de las poblaciones del parásito en todas las parcelas estudiadas. Se distingue de forma general una cierta relación entre el número de centros de agregación existentes y el promedio de muérdagos detectados en cada caso. Mayor cantidad de centros de agregación se relacionan con mayor densidad del parásito. Que fuera posible observar en cada uno de los mapas realizados la presencia de los centros de agresión bien definidos, permite afianzar lo hallado con los índices del SADIE, como se mencionó anteriormente.

Los numerosos focos de agregación detectados en cada caso, corroboran particularmente lo detectado por el índice $\mathrm{J}_{\mathrm{a}}$. En todos los casos los centros de agregación del parásito se hallaron repartidos más o menos por toda la parcela experimental. Habría que determinar en estudios posteriores si la altitud y la ubicación de las parcelas tienen algún efecto sobre el comportamiento espacial detectado en las poblaciones del muérdago enano.

De manera general en los mapas elaborados con el método del Krigeado Ordinario se observó que la superficie libre de infestación disminuyó conforme la densidad media era mayor. De esta manera se detectó que en las parcelas 3 y 5 fue donde existió la menor superficie sin infestación, por el contrario, en las parcelas 9 y 10 la superficie no infestada

Tabla 4. Porcentaje de superficie infestada y no infestada por el muérdago enano MAPAS ELABORADOS CON EL KRIGEADO ORDINARIO

\begin{tabular}{lcc}
\hline PARCELAS & \% INFESTADO & \% NO INFESTADO \\
\hline & & \\
P1 & 82,6 & 17,4 \\
P2 & 75,8 & 24,2 \\
P3 & 94,1 & 5,9 \\
P4 & 87,1 & 12,9 \\
P5 & 92,9 & 7,1 \\
P6 & 75,1 & 24,9 \\
P7 & 69,5 & 30,5 \\
P8 & 70,1 & 29,9 \\
P9 & 65,4 & 34,6 \\
P10 & 64,9 & 35,1 \\
\hline
\end{tabular}



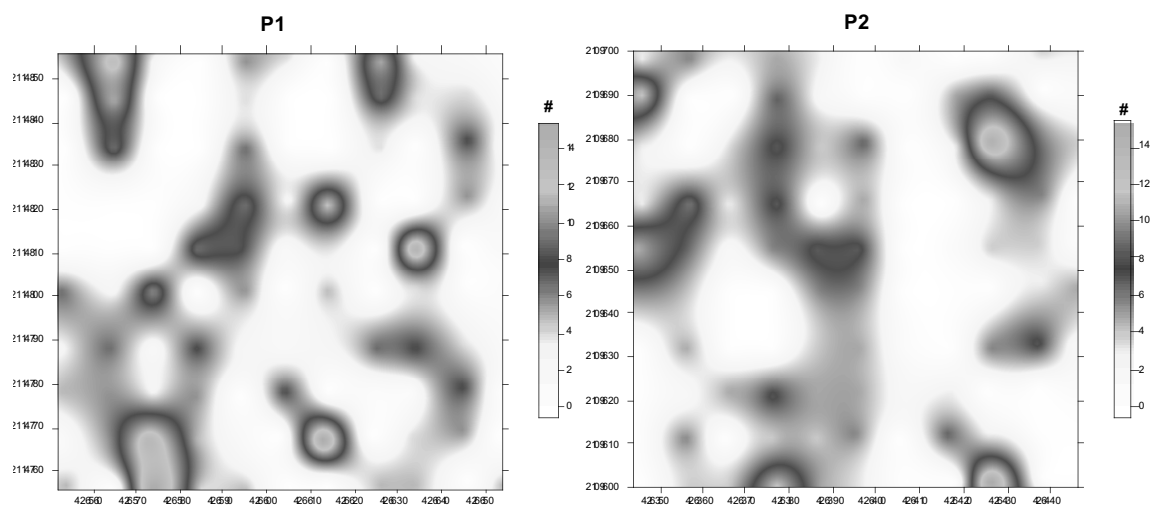

P3
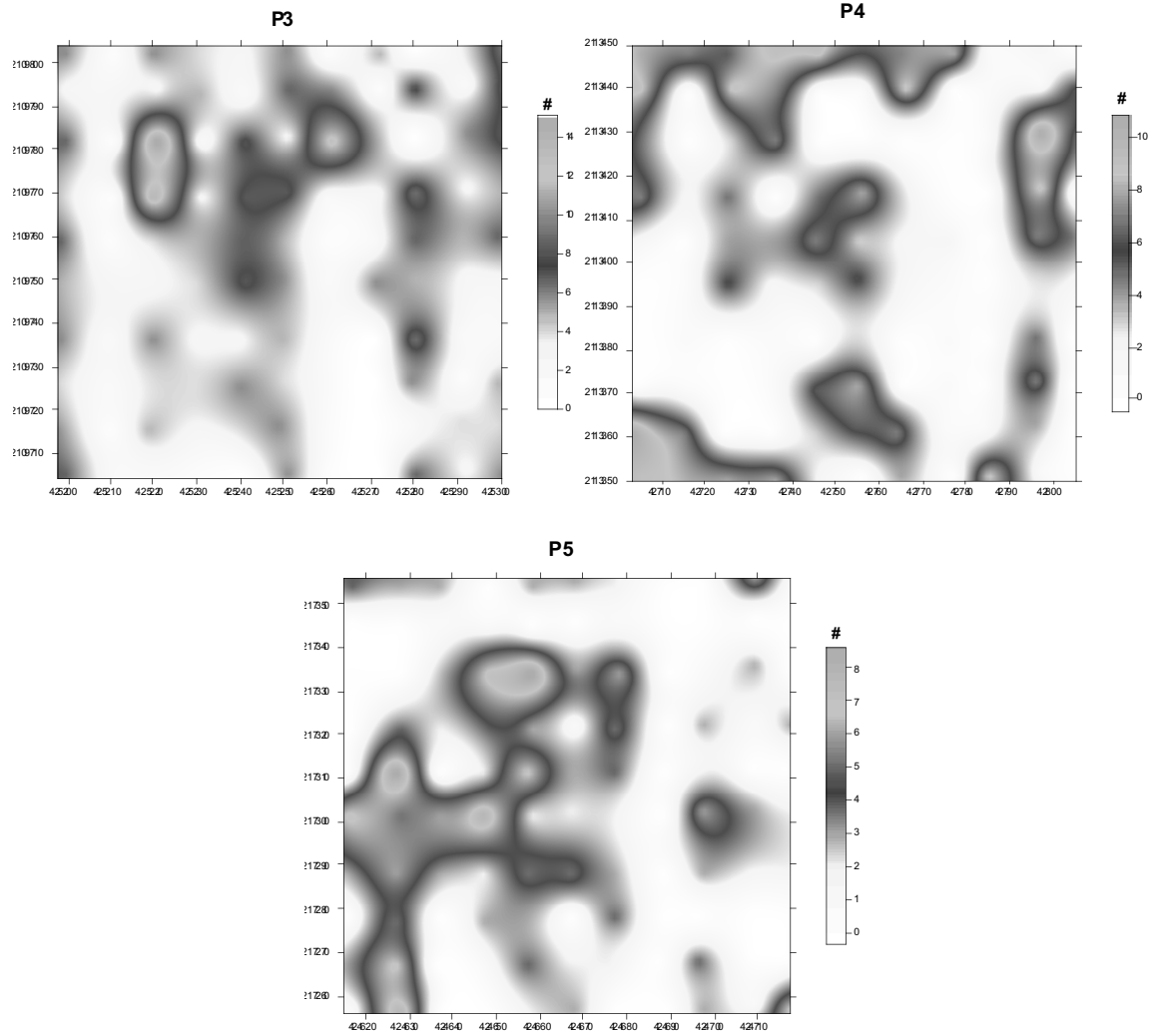

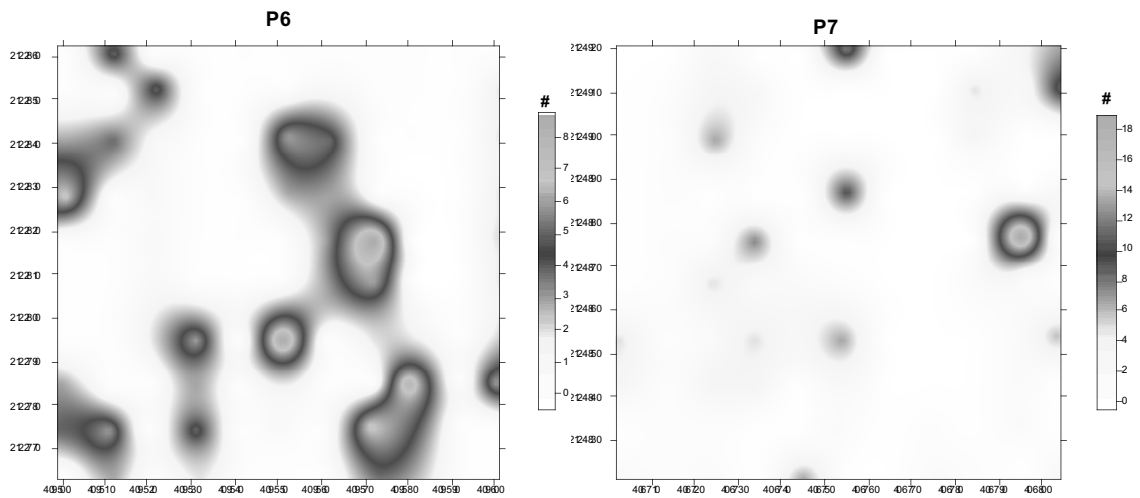

P8

P9
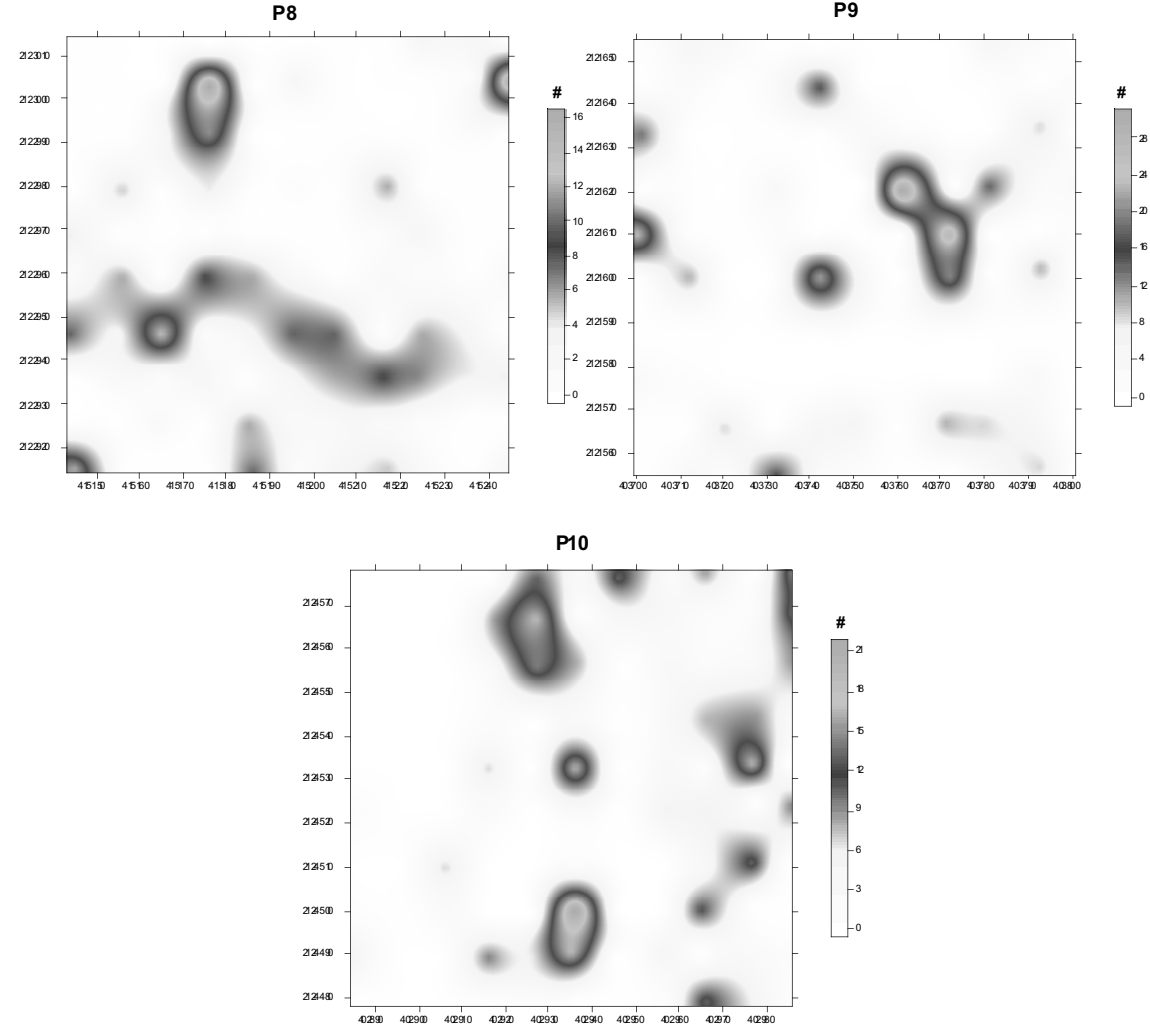

Figura 1. Mapas de densidad de las poblaciones del muérdago enano elaborados con antelación con el método del Krigeado Ordinario, para cada una de las parcelas experimentales. 
fue mayor. En el tabla 4 se detallan los resultados obtenidos del porcentaje de superficie infestada. En el caso de los mapas el porcentaje de superficie sin infestar se halló en el rango entre $35,1 \%$ y $5,9 \%$ del área total con un valor medio de $22,25 \%$. De manera general se encontró que a mayor densidad menor superficie sin infestar. Lo descrito resulta interesante, ya que en ningún caso la infestación provocada por el parásito, alcanzo $100 \%$. Lo anterior resulta de gran relevancia en el manejo integrado del muérdago enano, ya que permitiría realizar tácticas de control sobre las áreas específicas de infestación.

\section{Estabilidad espacial y temporal}

En la tabla 5 se concentraron los resultados de la comparación entre los mapas de la distribución espacial de las poblaciones del parásito, en cada parcela estudiada. Para llevar a cabo la comparación entre los diferentes mapas se utilizó el índice $I_{m}$ del SADIE; si los valores de este índice eran mayores que 0 , esto indicaba que no existía diferencia significativa entre los mapas comparados.

Una vez realizadas las 45 comparaciones posibles entre los mapas elaborados de las respectivas parcelas de

Tabla 5. Índices de asociación de distribución espacial comparando parcelas con muérdago enano.

\begin{tabular}{|c|c|c|c|c|c|}
\hline $\begin{array}{l}\text { PARCELAS } \\
\text { COMPARADAS }\end{array}$ & $\mathrm{I}_{\mathrm{M}}$ & $\begin{array}{c}\text { PARCELAS } \\
\text { COMPARADAS }\end{array}$ & $\mathrm{I}_{\mathrm{M}}$ & $\begin{array}{c}\text { PARCELAS } \\
\text { COMPARADAS }\end{array}$ & $\mathrm{I}_{\mathrm{M}}$ \\
\hline 1 vs. 2 & $-0,16$ & 2 vs. 9 & $-0,32$ & 5 vs. 6 & $-0,22$ \\
\hline 1 vs. 3 & $-0,35$ & 2 vs. 10 & $-0,39$ & 5 vs. 7 & $-0,88$ \\
\hline $1 \mathrm{vs} .4$ & $-0,22$ & 3 vs. 4 & $-0,67$ & 5 vs. 8 & $-0,38$ \\
\hline 1 vs. 5 & $-0,76$ & 3 vs. 5 & $-0,53$ & 5 vs. 9 & $-0,11$ \\
\hline 1 vs. 6 & $-0,31$ & 3 vs. 6 & $-0,14$ & 5 vs. 10 & $-0,40$ \\
\hline 1 vs. 7 & $-0,65$ & 3 vs. 7 & $-0,69$ & 6 vs. 7 & $-0,33$ \\
\hline 1 vs. 8 & $-0,72$ & 3 vs. 8 & $-0,12$ & 6 vs. 8 & $-0,51$ \\
\hline $1 \mathrm{vs} .9$ & $-0,95$ & 3 vs. 9 & $-0,44$ & 6 vs.9 & $-0,92$ \\
\hline 1 vs. 10 & $-0,27$ & 3 vs. 10 & $-0,95$ & 6 vs. 10 & $-0,80$ \\
\hline 2 vs. 3 & $-0,54$ & 4 vs. 5 & $-0,28$ & 7 vs. 8 & $-0,26$ \\
\hline 2 vs. 4 & $-0,70$ & 4 vs. 6 & $-0,57$ & 7 vs. 9 & $-0,19$ \\
\hline 2 vs. 5 & $-0,94$ & 4 vs. 7 & $-0,93$ & 7 vs. 10 & $-0,97$ \\
\hline 2 vs. 6 & $-0,53$ & 4 vs. 8 & $-0,28$ & 8 vs. 9 & $-0,37$ \\
\hline 2 vs. 7 & $-0,74$ & 4 vs. 9 & $-0,76$ & 8 vs. 10 & $-0,20$ \\
\hline 2 vs. 8 & $-0,66$ & 4 vs. 10 & $-0,48$ & 9 vs. 10 & $-0,26$ \\
\hline
\end{tabular}

Valores de Im > 0 indican asociación espacial 
estudio, de acuerdo con el índice $\mathrm{I}_{\mathrm{m}}$, no se halló asociación espacial alguna entre las comparaciones realizadas. Esto indica que no fue posible detectar una estabilidad espacio-temporal de las poblaciones de la plaga.

\section{DISCUSIÓN}

\section{Estadística Clásica}

Los índices de dispersión utilizados en este trabajo mostraron un escenario general de agregación en el comportamiento espacial del muérdago enano. Aunque el índice de dispersión (cociente media-varianza) y el índice de Green mostraron resultados similares en cuanto a la detección del agregamiento, la fuerza de dicha agregación la detectaron de diferente manera. En general el índice de Green encontró un mayor número de agregaciones leves. Si bien este índice es presentado como el más adecuado de los índices de dispersión, ya que es independiente del número total de individuos capturados y del valor medio de su abundancia, no es independiente del tamaño de la muestra (De los Santos, 1982). Por su parte, el índice de dispersión (cociente varianza-media) al utilizar la varianza para evaluar el grado de agregación presenta graves inconvenientes, ya que depende directamente de la abundancia (Leveche, 1972). Lo que hará que poblaciones con densidades medias altas presenten valores altos del índice, tal hecho limita en gran medida la fiabilidad del resultado obtenido.

El uso de distribuciones estadísticas presentó resultados similares a los de los índices de dispersión, con un ajuste casi general a la distribución Binomial Negativa. Dicho modelo tiene una larga tradición en la literatura ecológica para explicar el comportamiento espacial de los organismos (Pielov, 1977).
Asociado a la distribución Binomial Negativa tenemos el parámetro $k$, que ha sido muy utilizado como otro índice de agregación (Southwood, 1978 y Taylor, 1984). Lo valores de $k$ obtenidos en este trabajo se encuentran dentro del rango de valores generales para organismos vivos. Este índice, sin embargo, está influenciado por el tamaño de la muestra y de la unidad de muestreo (Cole, 1946; Morris, 1954). Si se logran mantener constantes estos factores, este índice proporciona una media muy útil del nivel de agregación de una población, siendo a la vez muy sensible al tipo de hábitat $y$ al estadío de desarrollo de las poblaciones (Hairston, 1959; Waters, 1954).

Los métodos estadísticos clásicos utilizados para establecer la distribución espacial de las poblaciones de organismos vivos tienen deficiencias y limitaciones. Tales deficiencias se han puesto de manifiesto en el presente trabajo. En muchas ocasiones los resultados obtenidos con ellos parecían ser aparentemente contradictorios, en otros casos no era posible ajustar los modelos de distribuciones espaciales y en otras circunstancias se ajustaban los datos a los dos modelos de distribución. A ello hay que añadir otra gran limitación de estos métodos, como es que no tienen en cuenta la naturaleza bidimensional de las poblaciones, es decir, la localización exacta de los individuos muestreados. Por consiguiente, no utilizan toda la información espacial disponible, lo que limita su alcance.

La distribución espacial agregada del muérdago enano fue estudiada por Gutiérrez (1970) y Andrade (1981); en ambos casos los autores utilizaron distribuciones espaciales en sus estudios y registraron algunos problemas con el ajuste estadístico correspondiente, como sucedió en este estudio. Rodríguez (1985) y Hernández Montiel (1999) deter- 
minaron el mismo comportamiento espacial del parásito utilizando diversos índices de dispersión y encontraron asimismo, problemas en la determinación de la distribución espacial del muérdago enano.

\section{Análisis Espacial por Índices de Distancia (SADIE)}

Este tipo de índices tienen la gran ventaja sobre los estudiados con anterioridad, que tiene en cuenta la localización espacial de la muestra, además de ser más intuitivos y biológicos que los índices no espaciales (Perry, 1995a,b). Los resultados obtenidos con estos índices $\left(\mathrm{l}_{\mathrm{a}} \mathrm{y} \mathrm{J}_{\mathrm{a}}\right)$ mostraron en todos los casos una estructura espacial en agregados de las poblaciones del muérdago enano, por lo que demuestran una mayor eficacia que los índices no espaciales. El índice $J_{a}$ también indica si la estructura espacial agregada se encuentra dispuesta en uno o varios centros de agregación. En este trabajo se encontró en la mayoría de los casos la existencia de varios centros de agregación.

También en el caso de la utilización del SADIE para establecer el tipo de distribución de este parásito sería interesante, en trabajos posteriores, analizar el patrón espacial con datos a diferentes escalas y a través de diversos periodos de tiempo, tal y como sugieren de forma general para el comportamiento espacial de organismos vivos Perry (1998), Ferguson et al., (2000) y Thomas et al., (2001). De esta manera se podrían comparar los resultados obtenidos y conocer con mayor detalle, en este caso, la distribución del muérdago enano y las bondades del método. Hay que destacar el hecho de que las diferentes altitudes en las cuales fueron establecidas las parcelas de estudio y el diámetro del tronco del pino, no influyeron en el establecimiento de la agregación por parte de las poblaciones de muérdago enano, tal y como se aprecia en los mapas.

Aunque no se determinó estadísticamente, se pudo observar en campo que, tomando en cuenta que el diámetro del tronco de los pinos está en relación con su edad, el parásito no parece tener preferencias de infestación en cuanto a la edad y talla del pino. Lo anterior resulta interesante, ya que Gutiérrez (1970) señala que la infestación del muérdago enano influye sobre el desarrollo en grosor del fuste del pino, por lo tanto, el ataque a pinos jóvenes reduciría el desarrollo posterior de estos árboles. Tomando en cuenta que una parte considerable de pinos muestreados en este estudio eran relativamente jóvenes, el impacto sobre su desarrollo tendrá gran importancia dentro del contexto ambiental del PNNT. La anterior aseveración habría que corroborarla estableciendo un estudio en el que se demostrara estadísticamente que el parásito infesta de forma generalizada sin importar edad y talla del pino.

Cabe señar que en la ladera sur del Parque Nacional Nevado de Toluca (cuenca del Balsas), se han registrado resultados similares en cuanto a la distribución espacial del muérdago enano; en el caso mencionado se utilizaron también los índices del SADIE (Ramírez-Dávila y González-Andujar, 2006). Con lo anterior se puede afirmar que el comportamiento espacial de este parásito en la totalidad del Parque Nacional Nevado de Toluca es agregado y distribuido en varios centros de agregación.

Los índices del SADIE se han utilizado con éxito en otros estudios para establecer la distribución espacial de organismos biológicos: Ferguson et al., 2000; Thomas et al., 2001; Winder et al., 2005; Alexander et al., 2005; Conrad, et 
al., 2006 y Ramírez-Dávila y GonzálezAndujar, 2006.

\section{Superficie infestada}

Pese a que el muérdago enano se encontraba en condiciones óptimas para su desarrollo, el porcentaje de infestación alcanzado en las diferentes parcelas de estudio nunca llegó a $100 \%$. Por lo tanto, las acciones de control deberán ser dirigidas sobre zonas específicas de infestación y no sobre la suposición de una colonización de $100 \%$ por parte del parásito, tal y como proponía en su momento González (1989), con la utilización de herbicidas selectivos. Es importante resaltar que, contando con las áreas específicas de infestación de este parásito sobre la zona de estudio, se podrían establecer programas de manejo de este problema sanitario del PNNT en su ladera norte, utilizando técnicas de precisión, como por ejemplo la adecuación de las propuestas sugeridas por Fleischer et al., 1999. Con lo anterior se podrían reducir los costos económicos y medioambientales, al reducir el uso de herbicidas selectivos en el control de esta plaga.

\section{Estabilidad espacial y temporal a corto plazo}

Se esperaba que existiera alguna estabilidad espacio-temporal en las poblaciones de muérdago enano, pero los resultados indicaron lo contrario. Habría que establecer si la altitud o la edad de los árboles influye en la manera de agregarse por parte de las poblaciones del parásito, de ser así, ésto explicaría por qué la manera de establecerse los agregados dentro de las parcelas de estudio fue diferente. Otra posibilidad podría ser, que el tamaño de las parcelas experimentales no permite detectar similitudes entre la forma de agregarse de las poblaciones de muérdago enano, por lo tanto, estudios posteriores podrían explorar la posibilidad de aumentar el tamaño de las parcelas para determinar si se puede detectar o no esa estabilidad. Como se mencionó anteriormente, la distribución agregada del parásito ya fue estudiada por Gutiérrez (1970), Andrade (1981), Rodríguez (1985) y Hernández (1994). Sin embargo, estos autores reportaron problemas con los métodos de Estadística No Espacial. En este estudio, el SADIE permitió sin lugar a dudas determinar el comportamiento agregado de las poblaciones del muérdago enano.

\section{CONCLUSIONES}

1. Los métodos no espaciales presentaron serias deficiencias y limitaciones al tratar de determinar el comportamiento de las poblaciones de muérdago enano.

2. Los análisis con SADIE presentaron un comportamiento espacial en agregación, con las poblaciones distribuidas en varios focos de concentración.

3. Los índices $\mathrm{I}_{\mathrm{a}}, \mathrm{J}_{\mathrm{a}}$ e $\mathrm{I}_{\mathrm{m}}$ demostraron ser eficientes en la determinación de la estructura espacial de las poblaciones de muérdago enano.

4. No se logró detectar estabilidad espacial y temporal en las poblaciones de la plaga.

5. Se identificaron áreas libres de infestación que permitían en su momento una aplicación dirigida de las medidas de control establecidas para este parásito.

\section{REFERENCIAS}

Alexander, C.J., J.M. Holland, L. Winder, C. Wolley \& J.N. Perry. 2005. Performance of sampling strategies in the 
presence of known spatial patterns. Annals of Applied Biology, 146: 361-370.

Alston. R. 1996. Statistical analisis of animal populations. Thesis PhD thesis, University of Kent. UK.

Andrade, E. V. 1981. Evaluación de efectos de muérdago enano (Arcetobium globosum Hawk Wiens, y $A$. vaginatum Wild) en rodales de Pinus hartwegi Lindl.

CEPANAF, UAEM y BIOCENOSIS, 1999. Programa de Manejo del Parque Nacional Nevado de Toluca.

Childs, W. I. y W. L. Edgron. 1976. Dwarf mistletoe effect on ponderosa Pine, growth and trunk form. Forest Science. 13 (2): 23-31.

Cole, L. 1946. A theory for analyzing contagiously distributed populations. Ecology. 27: 329 -341.

Conrad, K. F., J.N. Perry, I.P. Woiwod \& C.J. Alexander. 2006. Large scale emporal changes in spatial pattern during declines of abundance an occupancy in common moth. Journal of Insect Conservation, 10: 53-64.

De los Santos, A. C. Montes y L. RamírezDíaz. 1982. Modelos espaciales de algunas poblaciones de coleópteros terrestres en dos ecosistemas del bajo Guadalquivir (S.W. España). Mediterránea Ser. Biol. 6: 65-92.

Ferguson, W., Z. Klukowski, B. Walczak, J. Perry, M. Mugglestone, S. Clark, y I. Williams. 2000. The spatio- temporal distribution of adult Ceutorhynvhus assimilis in a crop of winter oilseed rape in relation to the distribution of their larvae and that of the parasitoid Trichomalus perfectus. Entomol.
Experimentalis et Applicata. 95: 161-171.

Fleischer, J., E. Blom y R. Weisz. 1999. Sampling in Precision IPM: When the objetive is a map. The American Phytopathological Society. 89 (11): 115-118.

Gill, L.S. y F. G. Hawksworth. 1961. The Mistletoe: a literature review U.S.D.A. Tech. Bull. No. 1242:87.

González, G. J. F. 1989. Biología y distribución de los muérdagos enanos en la unidad de Administración Forestal Tepehuanes.10-56.

Gutiérrez, R. R. M. 1970. Efectos de parásitos de muérdago enano (Arceuthobium sp.) sobre el desarrollo en grosor del fuste de Pinus montezumae Lamb. y Pinus hartwegii Lindl., en el cerro Telapon, Estado de México. INIF. (México). Boletín técnico. No. 34:22.

Hairston, N. G. 1959. Species abundance and organisation. Ecology. 40: 404-416.

Hawksworth, F. G 1961. Dwarf mistletoe of ponderosa Pine in the south west. U.S.D.A. Technical Bull No. 1246: 72.

Hernández Montiel, V. 1999. Evaluación de la infección causada por los muérdagos Struthanthus deppeanus (Cham \& Schlecht.) Blume y Arcetobium gilli subsp. Nigrum Hawks \& Wiens en pino de la Sierra Negra de Puebla. Tesis de licenciatura. Universidad Autónoma de Chapingo, $93 \mathrm{pp}$.

Korie, S., J. Perry, M. Mugglestone, S. Clark, C.F. Thomas y R. Mohamad. 2000. Spatio temporal association in beetle and virus count data. Journal 
of Agricultural, Biological y Environmental Statics. 5: 214-239.

Korstian, C.F. y Long. 1922. The Western Yellow Pine mistletoe. U.S.D.A. Bull No. 1112:35.

Leveche, C.H. 1972. Mollusques benthiques du lac Tchad: ecologie, etude des peuplements et estimation des biomasses. Cah. ORSTOM, ser Hidrobiol. 6(1): 3-45.

Morris, R. F. 1954. A sequential sampling technique for spruce bud worm eggs surveys. Canadien Journaly Zoologic 32: 302-313.

Perry, J. 1995a. Spatial aspects of animal and plant distribution in patchy farmland habitat. In: Ecology and Integrated Farming Systems. D. M. Glen, M. A. Greaves, H. M. Anderson (eds.). Chichester, England. Wiley. pp. 221-242.

Perry, J. 1995b. Spatial Analysis by distance indices. Journal of Animal Ecology. 64: 303-314.

Perry, J. 1998. Measures of spatial pattern for counts. Ecology. 79(3): 1008-1017.

Perry, J. y Hewitt, M. 1991. A new index of aggregation for animal counts. Biometrics. 47: 1505-1518.

Perry, J. y Klukowsky, Z. 1997. Spatial distributions of counts at the edges of sample areas. VI Conferencia de la Sociedad de Biometría. Córdoba, España, pp. 103-108.

Pielov, E. 1977. Mathematical Ecology. John Wiley \& Sons. Inc. 386 pp.

PROTINBOS, Protectora e Industrializadora de Bosques. 1972. Datos de Evaluación Forestal, Estudio Daso- nómico, Secretaría de Desarrollo Agropecuario, Metepec, Gobierno del Estado de México.

Ramírez-Dávila, J. F. y J. L. GonzálezAndújar. 2006. Modelización y mapeo de la distribución espacial del muérdago enano (Arceuthobium sp.) en la ladera sur del Parque Nacional Nevado de Toluca. Geografía Agrícola 38: 19-32.

Rodríguez, A. 1985. Infestación del muérdago enano Arceuthobium vaginatum (Hill) Presl., en el repoblado de Pinus hartwegii Lindl. del Parque Nacional Zoquiapan, Estado de México, Biol. Téc. Núm. 122. Instituto Nacional de Investigaciones Forestales, SARH, México.

Ross, C. J. S. 1987. Maximum likelihood program. Rothamsted Experimental Station Harpenden. UK.

Samper, F. J. y J. Carrera. 1990. Geoestadística: aplicaciones a la hidrología subterránea, CIMNE.

Sánchez-González, A. 2008. Una visión actual de la diversidad y distribución de los pinos de México. Madera y Bosques 14(1): 107-120.

Sokal, R. y F. Rohlf. 1995. Biometry: The Principles and Practice of Statistics in Biological Research. 3a ed., Freeman, New York. 356 pp.

Southwood, T. R. 1978. Ecological Methods, $2^{\mathrm{a}}$ ed., Wiley/Halsted. New York. 524 pp.

Taylor, L. 1984. Assessing and interpreting the spatial distributions of insect populations. Annual Review of Entomology 29: 321-357.

Thomas, G; L. Parkinson, K. Griffiths, G. Fernández y J. Marshall. 2001. 
Aggregation and Temporal Stability of Carabid Beetle Distributions in Field and Hedgerow habitats. Journal of Applied Ecology. 38: 100-116.

Valdivia S. J. de J. 1963. El muérdago enano (Arceuthobium sp.) de Michoacán. Tesis Profesional E.N.A. Chapingo, México.

Vega, R. E. 1976. El muérdago enfermedad de bosques de coníferas y hojosas, bosques y fauna. XIII. 1 . Departamento de Divulgación Forestal. SARH. México, 49 pp..

Verduzco, J. 1976. Protección Forestal. Ed. Patena. 369 pp.
Waters, W. E. 1954. A quantitative measure of aggregation in insects. Journal Economic Entomology 52: 180-184.

Winder, L., C. J. Alexander, J.M. Holland, W.O.C. Symondson, J.N. Perry, \& C. Wolley. 2005. Predatory activity and spatial pattern: the response of generalist carabids to their aphid prey. Journal of Animal Ecology, 77: 443-454. espacial del muérdago enano (Arceuthobium sp.) en el Nevado de Toluca, México, utilizando el método del SADIE. Madera y Bosques 15(2): 93-112. 